\title{
La crise
}

de la Covid-19

et ses impacts

sur la présence

chrétienne

dans la société,

spécifiquement

en Belgique ${ }^{1}$

Lentier. Beaucoup de gens meurent et davantage encore tombent malades. En plus de cet impact physique direct de la covid-19, la santé mentale des personnes qui contractent la maladie se trouve affectée; elles deviennent anxieuses ou se sentent stigmatisées, voire exclues. Outre les effets directs de la maladie sur les personnes, il faut noter les nombreux effets liés aux mesures visant à empêcher la propagation du virus. L'observance de la distanciation physique, l'annulation de nombreux événements, l'interdiction de réunions, la fermeture à des degrés divers, même temporaire, des écoles, des magasins ou d'autres services ont un impact sur la société entière. De nombreuses personnes souffrent des effets du confinement dû à la covid-19. Citons-en quelques-uns: l'augmentation de la pauvreté,

\section{DOSSIER}

Par Annemie Dillen

Annemie Dillen est professeure à la KULeuven ; elle est membre de l'unité de recherche de théologie pastorale et empirique de la Faculté de théologie et de sciences des religions. Elle est également " extraordinary researcher " à I'Université du Nord-Ouest, en Afrique du Sud. Ses recherches en théologie portent sur les familles et les enfants, la violence domestique, l'éducation religieuse dans les familles, la théologie empirique, I'aumônerie, la religion vécue, la spiritualité et la théologie pratique fondamentale. Dernières publications :

Dillen, A. \& Gärtner, S.,

Discovering Practical Theology: Exploring Boundaries, Louvain, Peeters, 2020 ; Wolfteich C.E., Dillen A., Catholic Approaches in Practical Theology, Louvain, Peeters, 2016.

Faculty of Theology and Religious Studies

Sint Michielsstraat, 6

3000 Leuven

Belgium

annemie.dillen@kuleuven.be

1. Je remercie Bernard Kasimato et André Fossion pour la traduction en français. 
le moindre développement économique, davantage de violence domestique, les inquiétudes en matières éducatives, les difficultés sur le plan relationnel, l'augmentation des suicides et des problèmes psychologiques. Dans certains cas, les gens tirent un profit positif de cette situation de pandémie: par exemple, ils acquièrent plus de conscience de ce qui essentiel dans la vie, ils passent davantage de temps en famille, etc. Cependant, globalement, ces quelques aspects positifs ne doivent pas nous rendre aveugles aux difficultés que rencontrent les gens partout dans le monde depuis le début de 2020, en raison de la Covid-19.

Eu égard à tous les effets évoqués ici de la Covid-19, il peut paraître un peu léger de considérer l'influence de la covid-19 sur l’église ou sur la société sous l'angle religieux. Il y a cependant deux bonnes raisons pour lesquelles je pense qu'il est important de le faire. Tout d'abord, comme théologienne (de théologie pratique), intéressée par la position de l'Église et par le rôle du christianisme dans la société, je pense qu'il est de mon devoir de me concentrer sur mon expertise propre. Ensuite, je pars du présupposé que la religion est importante dans le monde et qu'une juste expérience et compréhension de la foi chrétienne vécue en communauté peuvent être libératrices et avoir des effets thérapeutiques sur la vie quotidienne de nombreuses personnes.

Dans cet article, j'essaierai de développer un modèle théorique sur l'impact que la crise de la covid-19 peut avoir sur la présence chrétienne dans les sociétés. Je me référerai, bien sûr, à mon expérience en Belgique, l'un des pays où la présence de la covid-19 a été très forte, a conduit à des mesures importantes (parfois appelées «semi-confinement) et a provoqué de nombreux décès, surtout de personnes âgées. Tous les services religieux ont été interdits par l'État du mois de mars au mois de mai 2020 et, de nouveau, en novembre et décembre 2020. À la fin de décembre 2020, les services religieux étaient à nouveau autorisés pour 15 personnes. La Belgique se caractérise par une forte prévalence historique du catholicisme. Je parlerai également pour ma part d'un point de vue catholique.

Le modèle théorique que j'explique dans cet article tente de donner une vision globale de l'impact de la covid-19 sur la présence religieuse chrétienne dans la société. Tous les différents aspects de ce modèle devront être étayés par des recherches empiriques sur les faits et hypothèses ainsi que par une réflexion théologique et herméneutique, plus approfondie, sur divers aspects. Le modèle global pourra aider le lecteur à voir la complexité et les nombreux aspects de l'impact de la covid-19 sur le plan religieux. Après une première partie descriptive et analytique, je réfléchirai théologiquement sur le sens des différentes interrelations que j'aurai dégagées. 


\section{L'impact de la Covid dans une double direction}

Les recherches empiriques quantitatives qui analysent la relation entre deux phénomènes commencent principalement par la question de base: quelle est la variable indépendante et quelle est la variable dépendante? En l'occurrence, devons-nous considérer la «présence religieuse dans la société » comme une variable dépendante ou indépendante? La covid-19 est-elle le facteur qui a un impact sur la religion ou est-ce l'inverse ${ }^{2}$ ?

Les réponses peuvent être différentes. Certains articles décrivent l'impact de la covid-19 sur la religion ${ }^{3}$. D'autres études se concentrent, à l'inverse, sur l'impact de la religion sur la covid-194. Les chercheurs polonais Lukasz Sulkowski et Grzegorz Ignatowski ont décrit, sur la base d'entretiens avec des dirigeants de différentes églises chrétiennes, l'impact qui a été perçu de la covid-19 sur l'organisation des communautés religieuses locales ${ }^{5}$ et sur les mesures préventives qui ont été adoptées. Les chercheurs néerlandais Paul Vermeer et Joris Kregting ont décrit comment des pratiques religieuses spécifiques (telles que les services liturgiques, en particulier dans le « Bible belt » protestant orthodoxe aux Pays-Bas) avaient une influence sur la façon dont le virus pouvait se propager parmi les gens. Ils ont trouvé une corrélation significative entre la fréquentation de services liturgiques spécifiques (et parfois aussi l'appartenance à une église) et les hospitalisations ou décès dus à la covid-19 dans certaines contrées des PaysBas. Dans un modèle global, nous avons besoin d'une flèche allant de la « covid-19 » à la religion, et une autre qui va de la religion à la covid-19. Les deux variables ont une incidence l'une sur l'autre.

Il faut cependant se demander maintenant comment, dans ce modèle global, on décrit, d'une part, la variable « covid-19 » et, d'autre part, « la présence de la religion dans la société ». La covid-19 inclut ici le virus et la maladie, la propagation du virus, son impact sur le corps (la maladie/ le décès/les symptômes/la contagiosité...) et sur l'âme (santé mentale, questions existentielles comme l'anxiété...). La «présence de la religion dans la société » désigne, dans cet article, la manière dont les églises chré-

2. Paul Vermeer, The Challenges of the Covid-19 Pandemic in Doing Empirical Studies in Spirituality, Theology and Religion (Webinaire du 16 décembre 2020, organisé par Center for Empirical Studies in Spirituality, Theology and Religion aux Philippines).

3. Par exemple, Lukasz Sulkowski, Grzegorz IgNATowski, "Impact of Covid-19 Pandemic on Organization of Religious Behaviour in Different Christian Denominations in Poland ", dans Religions, 11, 5, 2020, p. 1-15, DOI : org/10.3390/rel11050254.

4. Par exemple, Paul Vermeer, Joris Kregting, " Religion and the Transmission of Covid-19 in The Netherlands », dans Religions 11, 8, 2020, p. 1-12, DOI : org/10.3390/rel11080393.

5. Lukasz SulkowsKi, Grzegorz Ignatowski, «Impact of COVID-19 Pandemic »; Voir aussi Nadia FadIL et al., « Religion and Covid-19. Anthropology of Religion », en ligne sur le blog de Nadia Fadil et ses étudiants : blog.associatie.kuleuven.be/anthropology_of_religion_/ (consulté le 5 février 2021). 
tiennes fonctionnent: comment elles rassemblent les gens, comment elles soutiennent les personnes dans la société et comment elles-mêmes sont perçues dans la société au sens large. Les deux variables majeures peuvent ainsi être qualifiées de multidimensionnelles.

Avec cette description large des deux variables principales, de nombreux exemples d'impacts dans les deux directions peuvent être décrits. Par exemple, les règles sur la distance physique afin de prévenir la covid-19 ont un impact sur la manière dont les professionnels religieux (tels que les aumôniers des hôpitaux) peuvent aider les patients et leurs familles. Dans le contexte belge, les expériences des aumôniers d'hôpitaux pendant la première période du semi-confinement (de mars-mai 2020) étaient très diverses: certains n'étaient pas autorisés à voir les patients atteints de la covid-19, mais pouvaient continuer à voir d'autres patients. Ils pouvaient soutenir les membres de la famille des patients de la covid-19 et entretenir des contacts téléphoniques avec ces patients eux-mêmes. Ils étaient présents pour l'accompagnement du deuil, pour des rituels et pour le soutien spirituel du personnel dans les hôpitaux. D'autres étaient à peine impliqués dans le fonctionnement quotidien de l'hôpital. Plus tard, de nombreux aumôniers ont également été autorisés à rendre visite aux patients atteints de la covid-19. Ils devaient cependant prendre toutes sortes de précautions. La majorité des aumôniers ont manifesté une créativité énorme et ont démontré la valeur de leur fonction ${ }^{6}$. Une recherche internationale sur la façon dont les aumôniers d'hôpitaux ont perçu leur travail pendant la crise de la covid-19 au printemps 2020 a été menée par ERICH (Institut européen de recherche pour l'aumônerie dans les hôpitaux) ${ }^{7}$. Dans cette recherche, la covid-19 est considérée comme une variable indépendante qui a influencé le travail des aumôniers d'hôpitaux ainsi que la manière dont ils sont perçus par les hôpitaux. En même temps, on peut dire que le travail des aumôniers d'hôpitaux, leur manière de pratiquer leur religion et leur offre de soins spirituels aux patients ainsi qu'à leurs familles (par exemple, en priant ensemble, en écoutant leur angoisse et leur espérance, en les soutenant dans leur dimension spirituelle), ont pu avoir un impact sur l'expérience de la covid-19 elle-même. Bien que les recherches sur les résultats des soins spirituels dispensés par les aumôniers dans les hôpitaux soient limitées, elles indiquent cependant que l'aumônerie hospitalière a souvent des effets positifs sur la santé mentale des patients ${ }^{8}$. Ce pourrait

6. Zinvol verbonden in tijden van corona, en ligne : www.pastoralezorg.be/page/elisabeth-zinvol-verbonden-in-tijden-van-corona/0/\#block1120231 (consulté le 5 février 2021).

7. European Research Institute for Chaplains in Healthcare, International Survey of Chaplain Activity and Experience during Covid-19 Pandemic, en ligne : www.pastoralezorg.be/page/international-survey-covid-19/ (consulté le 5 février 2021) ; A. VAndenhoeck et al., « Thematic issue on Covid-19 and hospital chaplaincy », dans Journal of Pastoral Care and Counseling, 75, 1, 2021, p. X. 
être le cas pour la covid-19, car nous pouvons supposer, sur la base des expériences des aumôniers, que les patients et les membres de leur famille peuvent se sentir moins anxieux et plus optimistes lorsqu'ils reçoivent les soins spirituels d'un aumônier. De nombreux aumôniers ont expliqué comment les rituels et les prières — également « à distance » — étaient très appréciés des patients ainsi que des membres de leur famille.

\section{Médiation}

À partir d'études empiriques, nous sommes conduits à penser en termes de variables «indépendantes» et «dépendantes ». Et en ce qui concerne les variables « la covid-19 » et « la présence de la religion dans la société », il semble qu'elles peuvent être " dépendantes » et «indépendantes ». En d'autres termes, la direction de l'influence n'est pas fixe; il y a probablement une influence mutuelle. Les connaissances basées sur des études empiriques nous amènent à nous interroger sur l'explication de ces relations bidirectionnelles. Quels sont les facteurs de médiation, les points d'articulation entre « la covid-19 » et « la présence de la religion dans la société »? Il peut y avoir une influence directe entre les variables, mais il peut y avoir également des facteurs multiples de médiation. En particulier, les diverses représentations qui circulent peuvent jouer un rôle de médiation. Les idées des responsables religieux, des professionnels et des bénévoles dans les paroisses, comme aussi les opinions des croyants, ou encore les idées diffusées par les médias ou par les décideurs politiques du pays peuvent avoir une influence sur les situations. Illustrons cela par des exemples en nommant explicitement les agents concernés. L'idée, par exemple, qu'une véritable communauté religieuse est constituée par des rencontres personnelles en face à face et par une participation physique à l'Eucharistie pourrait empêcher les responsables de prendre des initiatives de services numériques le dimanche. L'idée selon laquelle la catéchèse de confirmation doit se faire en petits groupes avec des enfants assis à une table pourrait empêcher les catéchistes (le plus souvent des bénévoles) d'inviter les parents et les enfants à une « marche catéchétique » ou à des rencontres catéchétiques à la maison. Des personnes se demandent si un

religious coping, and religious problem solving styles : A randomized controlled study ", dans Journal of Religion \& Health, 47, 1, 2008, p. 57-69, DOI : https://doi.org/10.1007/s10943-0079131-4 ; A. VANDENHOECK, "Reflections on research and professionalization in healthcare chaplaincy in Europe ", dans E. Kelly, J. Swinton (éds), Chaplaincy and the Soul of Health and Social Care. Fostering Spiritual Wellbeing in Emerging Paradigms of Care, London, Jessica Kingsley Publishers, 2020, p. 132143 ; A. Snowden, I.J.M. Telfer, « Patient reported outcome measure of spiritual care as delivered by chaplains ", dans Journal of Health Care Chaplaincy, 23, 4, 2017, p. 131-155, DOI : org/10.1080/08 854726.2017.1279935. 
pasteur ou un prêtre peut assurer un service pastoral par téléphone ou par une voie numérique. Si oui, cette pratique peut avoir une influence sur la manière de l'Église d'aider les gens qui se débattent dans des problèmes existentiels comme la solitude ou des difficultés financières, etc. De toute évidence, ces idées sont également influencées par la manière dont les chefs religieux agissent et communiquent. Les médias et les décideurs politiques (le gouvernement, les bourgmestres...) ont également un rôle à jouer. En Belgique, la vie ecclésiale est souvent réduite à la messe. Des mesures strictes interdisant les messes y ont été prises durant la période de mars à mai 2020. En mai 2020, les médias ont fait état de façons nouvelles et créatives d'organiser à nouveau les sports en plein air pour les enfants. Le Conseil interdiocésain pour la pastorale familiale ${ }^{9}$ dont je suis présidente a suggéré aux médias de parler également des possibilités créatives de vivre la religion à la maison. Ainsi, avons-nous suggéré de contacter une famille où la mère donnait régulièrement des séances de "Godly Play ${ }^{10}$ » à ses quatre enfants. C'est à ce moment-là qu'un journal nous a avertis que les messes seraient bientôt à nouveau autorisées. Cet exemple montre bien comment les responsables des médias influencent la manière dont l'Église est représentée et se rend présente dans la société. Les idées des décideurs politiques jouent également un rôle. Dans certains pays, les églises sont restées ouvertes, alors qu'en Belgique, les offices religieux et les réunions dans les églises ont été interdits pendant longtemps. Il y avait des limitations strictes pour les funérailles et pour les rituels autour des mourants. Ces réglementations strictes en Belgique pourraient être tributaires de décideurs politiques qui ne considèrent pas la religion comme importante. Dans les discussions sur la « stratégie de sortie », lorsque les cas de covid-19 ont commencé à baisser, la religion n'a pas été longtemps une question à l'ordre du jour. Les idées sur la relation entre l'Église, l'État et la liberté de religion sont également en jeu ici. Toutes ces idées peuvent influencer la manière dont une église est présente dans la société.

La question n'est pas seulement de savoir de qui proviennent les idées ayant une fonction de médiation, mais de savoir quelles sont ces idées. Il n'est pas possible d'en donner un aperçu complet. Nous en avons déjà énoncé quelques-unes. Le plus important est de citer celles qui font débat. Une communauté digitale ou une expérience numérique est-elle aussi précieuse qu'une vie de communauté réelle ${ }^{11}$ ? Privilégier les expé-

9. www.gezinspastoraal.be. Ce conseil est compétent pour la Belgique néerlandophone.

10. www.godlyplay.be.

11. Pour d'autres questions théologiques liées à la Covid-19 et à l’Église, voir Frederike van OORSCHOT, «Präsent sein. Ekklesiologische Perspektiven auf das kirchliche Leben unter den Bedingungen des Infektionsschutzes und seiner Folgen ", dans Benjamin Held et al. (éds), Corona als Riss. Perspektiven für Kirche, Politik und Ökonomie, Heidelberg, Universitätsbibliothek Heidelberg, p. 73-89. 
riences en face à face et attendre qu'elles soient à nouveau possibles, est-ce la meilleure option? Faut-il mettre en place une alternative numérique? Une question théologique sous-jacente est ce qui compte le plus: le contenu de la foi, du rituel et de la liturgie ou la forme? La vie ou le digital? Ceux qui se concentrent sur le contenu sont assez flexibles dans leur réflexion sur la forme de la communication de la foi, sur le rituel ou sur la liturgie. Ils pourraient opter sans trop de difficultés pour des alternatives numériques ou d'autres "moyens anti-corona » d'être église. Ceux qui, au contraire, se concentrent sur la forme peuvent avoir plus de difficultés. Pour eux, au sein de l'Église catholique, le corps et les aspects matériels sont traditionnellement pris très au sérieux. Tous les sens jouent un rôle dans la célébration de la foi. La crise du coronavirus met en question tous ces aspects. Les communautés souples qui inventent des connexions numériques sont souvent heureusement surprises du résultat de leurs efforts. Certaines communautés d'Église ont découvert que de nombreux paroissiens assistaient à des services en ligne et que même des familles avec les enfants s'y joignaient. En temps normal, les pères sont souvent absents dans les églises, mais pendant la période de confinement, certains pères, avec leur partenaire et leurs enfants, ont participé à la maison aux services en ligne.

Des conflits ont été vécus pendant les périodes de confinement: quel est le rôle des prêtres/du diocèse/de l'évêque? Est-ce que chaque diocèse ou chaque paroisse décide seul si la liturgie sera diffusée en ligne ou pas? Ou s'agit-il de décisions communes? Ce qui s'est passé en Belgique pendant le confinement était similaire à ce qui se passait auparavant: une tentative de prendre des décisions communes, mais en même temps beaucoup d'initiatives individuelles, au niveau de la base et au niveau des diocèses. Les manières de s'organiser en Église peuvent donc constituer un facteur de médiation entre la covid-19 et le rôle de l’église dans la société.

Les perspectives théologiques sur le rôle de Dieu dans la crise de la covid-19 pourraient également influencer la relation entre la covid-19 et la place de l’Église dans la société. Certaines personnes » se sont mises à douter de l'amour de Dieu et de son pouvoir, comme c'est souvent le cas lors d'une crise existentielle. D'autres ont découvert la présence de Dieu dans la solidarité que les personnes ont manifestée ou dans l'appel éthique à changer d'attitudes et à prendre soin des autres comme de soi-même. D'autres personnes sont devenues plus sensibles aux questions existentielles profondes et ont commencé à chercher des réponses dans les traditions religieuses. Une recherche plus approfondie sur les représentations de Dieu, telles qu'elles sont exprimées par les croyants et les non-croyants dans le contexte de la Covid-19, et sur l'ouverture à l'Église, pourrait être 
très intéressante ${ }^{12}$. En plus des questions fondamentales sur la vie, sur les structures ecclésiales, sur les questions de pouvoir et de théodicée, l'une des interrogations les plus répandues concerne les questions éthiques fondamentales. Qu'est-ce qui a le plus de valeur: la santé et la sécurité, d'une part, ou la liberté et la relation, d'autre part? Pendant de nombreux mois, ces valeurs ont été en conflit. Bien que la liberté personnelle, la liberté de religion, les relations et la vie communautaire soient très appréciées dans de nombreuses églises, beaucoup de responsables religieux s'en tiendront aux règles et aux politiques gouvernementales en donnant la priorité à la santé et à la sécurité. Le choix cependant n'est pas facile, car la santé et la sécurité sont importantes, mais elles ne doivent pas cependant prendre la place du « sacré » absolu ou même devenir une religion ${ }^{13}$. Les dirigeants de l'Église sont mis au défi d'aider les personnes à vivre en relation et en communauté, avec liberté malgré les nombreuses restrictions, en offrant des alternatives créatrices de connexions et de rencontres. Diverses perspectives théologiques pourraient ainsi servir de médiation entre la covid-19 (et les mesures prises) et la présence de l'Église dans la société. Ces perspectives ne sont pas figées; elles pourraient changer en raison de l'évolution de la covid-19 ou en raison des pratiques concrètes de l'Église.

\section{Réflexions critiques de théologie}

En explorant ici la relation entre la covid-19 et la présence de l'Église dans la société et en repérant la médiation que la théologie peut exercer, j'ai également donné un aperçu de quelques aspects du contexte belge. À ce stade, une réflexion théologique plus approfondie sur le modèle que j'ai présenté ci-dessus serait nécessaire. Tout d'abord, il conviendrait de déployer deux points de vue critiques et complémentaires par rapport au modèle proposé. J'ai écrit que la relation entre la covid-19 et la présence de l'Église dans la société pouvait passer par des idées théologiques. C'est pourquoi j'ai insisté sur le fait que plus de recherches empiriques seraient nécessaires. En tant que théologienne pratique, j'ai des raisons supplémentaires d'ajouter une mise en garde par rapport aux idées. Car, si les idées peuvent servir de médiation dans la relation, il se peut aussi que les idées et croyances religieuses soient elles-mêmes les résultats des pratiques de l'Église fonctionnant comme des facteurs de médiation. La crise de la covid-19 a incité de nombreuses églises à organiser, par exemple, des acti-

12. Pour les questions de théodicée et de la Covid-19 dans l'éducation religieuse, voir www. kuleuven.be/thomas/page/coronavirus. 
vités intergénérationnelles en famille, en plein air (des promenades, par exemple, en préparation de la Noël ou de la fête de la Chandeleur). Ces nouvelles pratiques pourraient avoir une influence sur les idées des responsables de paroisse concernant les familles, concernant la communication religieuse ou concernant les possibilités et la pertinence de l'apprentissage intergénérationnel. Des expériences positives d'activités intergénérationnelles au sein de la petite "bulle familiale " pourraient conduire à une plus forte conviction que l'Église peut également atteindre les parents et pas seulement se concentrer sur les activités catéchétiques destinées aux enfants. Ce n'est qu'un exemple, mais il est clair que les pratiques et les idées interfèrent entre elles et qu'il serait trop facile de s'attendre à ce que chaque changement de pratique soit le résultat d'une réflexion approfondie ou d'hypothèses explicites. Il peut y avoir également une flèche directe qui va de la crise de la covid-19 vers les pratiques, les activités et la présence de l'Église, suivie d'un autre flèche qui va des pratiques vers les croyances, idées et hypothèses.

Un deuxième point de vue critique complémentaire sur le modèle présenté au point 3 concerne son objectif. Tel que je l'ai mis en œuvre ci-dessus, le modèle est simplement descriptif; il peut fonctionner comme un modèle théorique cohérent pour différentes études empiriques. En tant que théologienne, je suis cependant également intéressée par les questions normatives: "Qu'est-ce qui devrait être? » Afin de trouver un sens à la crise actuelle du coronavirus, de nombreux faiseurs d'opinion ont écrit sur les leçons qui peuvent être tirées de cette crise. Les gens espèrent plus de solidarité, plus d'attention pour le développement durable, plus de reconnaissance de la fragilité humaine, etc ${ }^{14}$. $\mathrm{D}^{\prime}$ autres suggèrent que cet espoir pourrait être vain et craignent que les choses, à l'avenir, continuent comme d'habitude, et probablement dans des conditions économiques et sociales pires que dans le passé. Nous pourrions également exprimer l'espoir que les Églises et les gens en général puissent apprendre quelque chose et que leurs idées puissent changer pour le mieux. Cette situation pourrait nous rappeler ce qui s'est passé après deux autres crises principales. Après l'holocauste, de nombreux théologiens ont appelé à un renouveau de la théologie. La crise des abus sexuels dans l'Église catholique a également incité les théologiens à un renouvellement de la théologie ${ }^{15}$. Cette analogie doit être considérée avec précaution pour deux raisons. Tout d'abord, tirer les leçons d'une crise réelle dans laquelle de nombreuses personnes souffrent,

14. Par exemple, Dirk Draulans, " Onze hoogmoed keert zich tegen ons », dans Knack, 4 mars 2020, p. 36-38; Hendrik Schoukens, «L'homme est et reste un être minuscule. Le coronavirus nous oblige à une leçon d'humilité », dans Knack, 21 mars 2020, p. X. 
peut être considéré comme une instrumentalisation de la souffrance des autres. La douleur, la souffrance elle-même, doivent être prises au sérieux et doivent être reconnues. Apprendre quelque chose ou espérer un changement semble toujours très ambigu: le changement espéré ne doit pas être invoqué pour minimiser la souffrance actuelle de nombreuses personnes. Deuxièmement, la crise de la covid-19 est quelque peu différente: bien que des idées générales (sur la manière dont les humains traitent la nature, sur le vivre ensemble, sur les conditions de vie et la pauvreté) aient pu avoir une influence sur le déclenchement de la crise de la covid-19, il est moins clair que, par exemple, dans le cas de l'holocauste ou de la crise des abus sexuels dans l'Église catholique, des idées théologiques spécifiques (sur les Juifs et les relations judéo-chrétiennes, ou sur le pardon, sur l'autorité dans l'Église et sur la sexualité) aient joué un rôle dans le développement de la crise. Il y a bien sûr des idées exprimées par certains dirigeants d'Églises à travers le monde, qui pourraient être très problématiques, comme l'idée selon laquelle Dieu protégerait les gens contre le virus-corona et que des mesures de protection n'étaient donc pas nécessaires ${ }^{16}$. Surtout au début de la crise, on a entendu de tels propos dans certains groupes religieux, bien qu'ils ne furent pas très présents en Belgique. Malgré ces mises en garde sur la façon dont la crise de la covid-19 pourrait défier la théologie, je voudrais exprimer mon espoir qu'au sein de l'Église catholique en Belgique et dans le monde, les théologiens poursuivront leurs efforts dans la lignée de Laudato si' et Fratelli tutti afin que la société puisse être un lieu où tout le monde peut s'épanouir et où le développement durable soit au cœur des préoccupations. Comme la covid-19 rend manifeste la position privilégiée d'une minorité et la situation difficile de beaucoup d'autres personnes, en particulier celles qui vivent dans la pauvreté, qui sont de santé fragile ou avancées en âge, j'espère que les concepts de la pensée sociale chrétienne comme "l'option pour les pauvres ", "la solidarité » et « la justice sociale » seront de plus en plus au cœur des réflexions théologiques et des pratiques ecclésiales.

Une troisième réflexion qui complète le point de vue descriptif des parties précédentes de cet article est une réponse à la question: que nous apprend le modèle descriptif avec toutes les flèches tel que je l'ai décrit plus haut?

Tout d'abord, une crise comme celle de la covid-19 est l'occasion d'apprendre la flexibilité, la réflexion proactive, l'innovation et la pensée « hors des sentiers battus ». D'un point de vue théologique, on pourrait peut-être dire que la tradition chrétienne est intrinsèquement flexible, 
car elle s'est toujours adaptée à des circonstances différentes ${ }^{17}$. Pour étayer cela, on se réfère parfois aux premières communautés chrétiennes, qui n'avaient pas toutes les structures et possibilités de se réunir et de célébrer ensemble comme les Belges les avaient avant mars 2020. Le christianisme ne repose pas sur une tradition fermée. Il serait intéressant, à cet égard, de mener une discussion sur le rôle actif que les dirigeants chrétiens devraient pouvoir jouer dans les circonstances présentes. Il est sage, dans ces circonstances, de plaider pour une forme concertée, au sein de l'Église, de représentation et d'intervention. Certes, l'Église, la vie chrétienne, la foi ne peuvent pas être «créées » et ne dépendent pas seulement des efforts humains. La confiance en la présence de Dieu, en l'Esprit de Dieu qui guide les gens dans leur vie quotidienne et le pouvoir de la foi sont plus importants. La « situation de crise » actuelle n'est donc pas simplement l'occasion à saisir pour un renouveau de l'Église. Cette situation de crise, en effet, est une perte. Elle est douloureuse. Et la souffrance de nombreuses personnes, physiquement et mentalement, doit être reconnue. Mais, d'un autre côté, dans cette rupture même, dans les «fragments » (Henning Luther) ${ }^{18}$, dans les éclats et les cicatrices que la crise occasionne, quelque chose de «saint » et des traces de Dieu pourraient être découverts.

Deuxièmement, la réflexion sur la relation entre la covid-19 et la présence de l'Église dans le monde nous montre combien les gens attendent de l'Église qu'elle continue à avoir un sens. Les bâtiments de l'église devaient peut-être rester fermés, mais beaucoup de gens espéraient de l'Église des formes de vie communautaire, une pastorale innovante et, dans de nombreux cas aussi, une voix prophétique et critique. Après les premiers mois de la crise, de nombreux catholiques et dirigeants d'Églises ont regretté de n'avoir pas fait entendre davantage leur voix, en protestant contre les règles qui laissaient les gens mourir seuls, ou contre la l'interdiction de tout contact empêchant les gens de dire au revoir à leurs proches avant de mourir. (En Belgique, les taux de mortalité les plus élevés ont été constatés au printemps 2020). Une position plus prophétique de l'Église catholique aurait pu être préférée, s'agissant notamment des soins adéquats à prodiguer aux personnes âgées ou s'agissant de l'attention à porter à tous et celles qui souffraient davantage de violence domestique et de pauvreté. Pour beaucoup de gens, l'Église semblait absente, en raison des règles en vigueur relatives au coronavirus, mais aussi en raison de son manque de parole publique. Des aumôniers d'hôpitaux, des pasteurs de paroisse, des

17. Par exemple, Erik Borgman, Alle dingen nieuw. Een theologische visie voor het $21^{\text {ste }} e e u w$, Utrecht, Kok, 2020.

18. H. Luther, Religion und Alltag. Bausteine zu einer Praktischen Theologie des Subjekts, Stuttgart, Radius-Bücher, 1992. 
chrétiens à titre personnel ont offert un énorme soutien aux gens. Mais ce soutien de la part de l'Église aurait pu s'étendre à beaucoup d'autres.

Troisièmement, le modèle présenté plus haut ne fait découvrir, en fait, qu'une partie du réel et montre à quel point la réalité est fragile, à quel point l'éthique est vulnérable et à quel point il faut éviter de voir des relations directes entre les problèmes et leur solution, entre la crise et la guérison. Il y a de multiples facteurs qui interviennent et donc, forcément, des réactions et des effets différents. Il importe d'éviter des explications trop faciles dès lors qu'une crise est résolue et que les gens manifestent de la résilience. Les cicatrices, la douleur et la souffrance des gens ne doivent pas être estompées par une pensée trop facile de la résurrection ou par l'idée que la religion répond à la complexité des situations ${ }^{19}$. La religion elle-même, en particulier le christianisme, fait partie de la complexité des choses et participe à la confusion, à la douleur et à la souffrance de tous.

Quatrièmement, la crise de la covid-19 fonctionne avant tout comme une loupe qui rend plus clair ce qui existait déjà. C'est vrai pour les bonnes choses: la solidarité, l'ouverture aux nouvelles initiatives créatrices, les soins, le soutien mutuel des églises et au sein de l’Église. Mais c'est également vrai pour les aspects plus sombres tels que les conflits, les abus de pouvoir et la négligence dans le domaine de la solidarité sociale. Ce peu d'attention, dans la société et comme dans l'Église, pour les soins aux personnes âgées, aux personnes victimes de violence domestique, aux enfants pauvres ou maltraités, n'est pas neuf. La crise du coronavirus a rendu ces déficits plus visibles.

J'espère que cette petite contribution a éveillé l'intérêt pour l'étude de la relation entre la Covid-19 et la religion, surtout dans une perspective qui souligne la complexité des choses et la bidirectionnalité des facteurs qui interviennent. De nombreux aspects sont difficiles à appréhender dans la recherche empirique. C'est pourquoi j'ai ajouté ici une réflexion, forcément limitée, de théologie critique. Le nombre de publications, de conférences, de séminaires sur la covid-19 et la religion augmente chaque jour ${ }^{20}$.

19. Judith Gruber, «Doing Theology with Cultural Studies. Rewriting History - Reimagining Salvation - Decolonizing Theology », dans Louvain Studies, X, 2019, p. 103-123.

20. Par exemple, Claudia GÄrTnER, Klima, Corona und das Christentum : Religiöse Bildung für nachhaltige Entwicklung in einer verwundeten Welt, Bielefeld, Transcript Verlag (Religionswissenschaft, 20), 2020 ; Paul M. Zulehner, Bange Zuversicht, Was Menschen in der Corona-Krise bewegt, Ostfildern, Patmos, 2021 ; Bert RoebBen, Volharden in de broosheid. Spiritualiteit in tijden van corona, Antwerpen, Halewijn, 2020. Voir les revues qui prévoient des numéros consacrés à la Covid-19 (à paraître en 2021), par exemple, Open Theology, Sociology of Religion, Journal of Pastoral Care and Counseling et Journal of the Academy of Religion. 
J'ai écrit cette réflexion surtout avec un énorme sentiment de gratitude. N'ayant pas souffert moi-même directement (physiquement) du virus, le fait d'avoir pu écrire cet article est un privilège. En même temps, je veux exprimer mon empathie à l'égard des personnes qui ont perdu des êtres chers, qui travaillent quotidiennement pour combattre le virus ou qui doivent prendre, chaque jour, des décisions très difficiles.

\section{The Covid-19 Crisis and its Impact on the Christian Presence in Society, and Especially in Belgium}

Empirical research about covid-19 and religions is growing. In this article, the author explains possible ways of researching the relationship between the covid-19 crisis and the presence of Christian churches in Belgium. She refers to possible mediations of theological ideas, which might influence how the covid-19 crisis and the presence of Christian churches in society are interrelated. As a practical theologian, she add critical theological reflections and illustrates the theoretical model she develops with examples of practices in Belgium. 